TRANSACTIONS OF THE

AMERICAN MATHEMATICAL SOCIETY

Volume 353, Number 5, Pages 1921-1936

S 0002-9947(01)02709-X

Article electronically published on January 4, 2001

\title{
ARITHMETIC DISCRIMINANTS AND MORPHISMS OF CURVES
}

\author{
XIANGJUN SONG AND THOMAS J. TUCKER
}

\begin{abstract}
This paper deals with upper bounds on arithmetic discriminants of algebraic points on curves over number fields. It is shown, via a result of Zhang, that the arithmetic discriminants of algebraic points that are not pullbacks of rational points on the projective line are smaller than the arithmetic discriminants of families of linearly equivalent algebraic points. It is also shown that bounds on the arithmetic discriminant yield information about how the fields of definition $k(P)$ and $k(f(P))$ differ when $P$ is an algebraic point on a curve $C$ and $f: C \longrightarrow C^{\prime}$ is a nonconstant morphism of curves. In particular, it is demonstrated that $k(P) \neq k(f(P))$, with at most finitely many exceptions, whenever the degrees of $P$ and $f$ are sufficiently small, relative to the difference between the genera $g(C)$ and $g\left(C^{\prime}\right)$. The paper concludes with a detailed analysis of the arithmetic discriminants of quadratic points on bi-elliptic curves of genus 2 .
\end{abstract}

Let $C$ be a curve defined over a number field $k$, and let $X$ be a regular model for $C$ over the ring of integers $R$ of $k$. In $\mathrm{V} 3$, Vojta proves that, for any $\epsilon>0$, all points $P \in C(\bar{k})$ of bounded degree satisfy the inequality

$$
h_{K}(P) \leq d_{a}(P)+\epsilon h_{A}(P)+O(1),
$$

where $A$ is any ample divisor on $C, h_{A}$ and $h_{K}$ are Weil heights associated with $A$ and the canonical divisor $K$, respectively, and $d_{a}(P)$ is the arithmetic discriminant of $P$. The arithmetic discriminant depends on the choice of a regular model $X$; it is defined as

$$
\frac{\left(H_{P} \cdot\left(\omega_{X / B}+H_{P}\right)\right)}{[k(P): k]},
$$

where $H_{P}$ is the arithmetic curve on $X$ corresponding to $P$ and $\omega_{X / B}$ is the sheaf of relative differentials of $X$ over $B=\operatorname{Spec} R$. The difference between arithmetic discriminants derived from different regular models is always bounded. Hence, (0.0.1) is not affected by one's choice of a regular model.

The arithmetic discriminant is related to $d(P)$, the normalized field discriminant of the field of definition of $P$, which is defined as

$$
d(P):=\frac{\log \left|N_{\mathbb{Q}}^{k} D_{k(P) / k}\right|}{[k(P): \mathbb{Q}]} .
$$

One may obtain $d_{a}(P)$ by adding to $d(P)$ terms corresponding to singularities of $H_{P}$ at finite places and $v$-adic distances between the conjugates of $P$ at infinite places $v$ (see [V2, Section 3]). The precise nature of the relationship between

Received by the editors November 30, 1999 and, in revised form, February 25, 2000.

2000 Mathematics Subject Classification. Primary 11G30, 11J25.

(C)2001 American Mathematical Society 
$d(P)$ and $d_{a}(P)$ is of great interest. Vojta's conjecture, which implies a variety of conjectured diophantine inequalities ( $\mathrm{V} 1,5 . \mathrm{ABC}])$, is that (0.0.1) still holds when $d_{a}(P)$ is replaced by $d(P)$ ([V 1, Conj. 5.2.6]).

At this time, little is known about the arithmetic discriminants of algebraic points in general. In $[\mathbf{S - T}$, it is suggested that it may be the case that on any curve of nonzero genus, the inequality 0.0 .1 is sharp, in the sense that, for any $\epsilon>0$, there are infinitely many $P$ of bounded degree for which $h_{K}(P) \geq d_{a}(P)-$ $\epsilon h(P)+O(1)$. This is currently only known, however, in the somewhat immediate case of curves that admit nonconstant morphisms to elliptic curves. On $\mathbb{P}^{1}$, there is a simple formula for $d_{a}(P)$ in terms of $h_{K}(P)$ ([V 2, Lemma 3.4 (d)], but the formula does not apply to curves of higher genus. In [S-T, Section 2], it is shown that the formula for $d_{a}(P)$ on $\mathbb{P}^{1}$ extends to a simple upper bound (though not an exact formula) that applies to all algebraic points of bounded degree on a curve of any genus. Here we give a precise description of $d_{a}(P)$ in terms of a certain height function of a point on $\operatorname{Pic}^{0}(C)$ related to $P$. It is difficult to gain complete control of the heights of the related points, but we are able to show that our original upper bound can be improved for points whose conjugates do not form the fiber of a map to $\mathbb{P}^{1}$. We are also able to construct examples of quadratic points on curves of genus 2 that have arithmetic discriminants which do not behave like rational points of curves of genus 1 or less. The techniques we use to show that certain points have small arithmetic discriminants are different from the ones used to produce points with small field discriminants in $[\mathrm{S}-\mathrm{T}$. Section 4]; the method of $[\mathrm{S}-\mathrm{T}$ involves producing points $P$ for which $d_{a}(P)$ may be large but the difference $d_{a}(P)-d(P)$ is also large. We hope to combine the techniques of $[\mathrm{S}-\mathrm{T}]$ with the techniques of this paper to produce families of points $P$ for which $d_{a}(P)$ is small and the difference $d_{a}(P)-d(P)$ is large. It seems conceivable (though by no means certain) that such points might have field discriminants $d(P)$ smaller than $h_{K}(P)$ and thus constitute counterexamples to Vojta's conjecture.

The arithmetic discriminant has other interesting properties besides its connection with the field discriminant and Vojta's conjecture. For example, it yields information about the behavior of the degrees of images of algebraic points under morphisms. Suppose that we have a nonconstant morphism $f: C \longrightarrow C^{\prime}$ between curves defined over a number field $k$. It is a basic property of the arithmetic discriminant that for all $P \in C(\bar{k})$ such that

$$
k(P)=k(f(P)),
$$

the bound $d_{a}(P) \leq d_{a}(f(P))$ holds ([V2, $\left.\left.3.4(\mathrm{e})\right]\right)$. This enables us to translate inequalities involving the arithmetic discriminant into statements about the behavior of points satisfying (0.0.2). Vojta uses (0.0.1) and the formula for $d_{a}(P)$ of points $P \in \mathbb{P}^{1}(\bar{k})$ to show that when $C^{\prime}=\mathbb{P}^{1}$, there are only finitely many points with degrees small relative to the genus of $C$ and the degree of $f$ that satisfy (0.0.2) ( Cor. 0.2]). Our extension of the formula for the arithmetic discriminant of algebraic points on $\mathbb{P}^{1}$ to an upper bound for arithmetic discriminants of points on curves of any genus enables us to show that Vojta's result still holds when the base curve $C^{\prime}$ has nonzero genus. Under certain circumstances, we can use our improvement of the bound for points that aren't obtained from rational points on $\mathbb{P}^{1}$ to give a better version of this result. Questions concerning points such that $f(P)=k(f(P))$ are closely related to questions about generalized versions of Hilbert's irreducibility theorem, which are taken up in $\mathrm{Tu}$. 
It also turns out that our result on point satisfying (0.0.2) yields a simple "arithmetic" proof of Castelnuovo's genus-inequality for curves that are covers of two other curves ( $\mathrm{A}-\mathrm{C}-\mathrm{G}-\mathrm{H}$, Chapter 8, Ex. C-1]) in the case where one of the two other curves is elliptic. Moreover, we are able to sharpen this genus-inequality when certain hypotheses hold. We do not know if there is a geometric proof of this improvement. In [S-T, Section 3], it was shown that the sharpness of Vojta's conjecture relates to the classical Severi problem for singular curves on surfaces, so in some sense the connection between arithmetic discriminants and the Castelnuovo genus-inequality fits into a pattern of connections between the work of Vojta on arithmetic surfaces and classical theorems for geometric surfaces.

We note that our results have a similar flavor to those of $[\mathrm{A}-\mathrm{H}]$, although the techniques of $\mathrm{A}-\mathrm{H}$ are entirely different from ours. In fact, Abramovich and Harris ask whether Vojta's result on points satisfying (0.0.2) admits a generalization such as ours ( $\mathrm{A}-\mathrm{H}$. Remark 1.2]). Furthermore, the work of Abramovich and Harris gives rise to an improvement on Castelnuovo's second genus-inequality that is quite similar to ours.

Let us now give a brief outline of this paper. We describe the terminology and notation of this note in the next section. We then proceed to show that the upper bound

$$
d_{a}(P) \leq h_{K}(P)+2 \nu h(P)+O(1),
$$

for any algebraic point $P$ of degree $\nu$, is an immediate consequence of the Arakelov adjunction formula and the structure theorem for the Arakelov-Picard group of an arithmetic surface. After that, in Proposition 2.3, we produce our generalization of Vojta's result on points satisfying 0.0 .2 . This allows us to give an arithmetic proof, in Corollary 2.5, of a special case of Castelnuovo's second genus-inequality for curves. In Section 3, we use an idea of Zhang ([Zh] to prove Corollary 3.3 which shows that many algebraic points $P$ of degree $\nu$ satisfy

$$
d_{a}(P) \leq h_{K}(P)+(2 \nu-2+\epsilon) h(P)+O(1)
$$

for any $\epsilon>0$. This bound yields an improvement of Proposition 2.3 in some cases; this improvement is presented in Proposition 3.4. We are then able to obtain a stronger version of Castelnuovo's second genus-inequality, under hypotheses, in Corollary 3.5. We conclude this note by looking at quadratic points on curves of genus 2. In particular, we construct a family of quadratic points on a bi-elliptic curve of genus 2 which possess arithmetic discriminants that behave differently than the arithmetic discriminants of pull-backs of rational points from an elliptic curve or the projective line.

\section{Preliminaries}

We need to introduce a bit of Arakelov theory in order to make all of our definitions precise. Let $C$ be a curve over a number field $k$. All curves in this paper will be assumed to be nonsingular, complete, irreducible, and defined over a number field, unless otherwise specified. Let $X$ be a regular model for $C$ over the ring of integers $R$ of $k$ (for a proof that one exists, see $\underline{\mathrm{Ar}}$ ). At the infinite places $\sigma$ of $k$ (which correspond to embeddings $\sigma: k \hookrightarrow \mathbb{C}$ ), we may endow $C \times_{\sigma} \mathbb{C}$ with an admissible Arakelov volume form (see [L 2, 4.3]). This allows us to find local and global arithmetic intersections of arithmetic divisors on $X$ as in $\mathrm{V} 3]$. 
An algebraic point $P \in C(k)$ gives rise to a horizontal divisor $H_{P}$ on $X$ (by taking the closure of the support of $P$ in $C$ ). We define the canonical height $h_{K}$ of a point $P \in C(k)$ to be

$$
h_{K}(P):=\frac{\left(H_{P} \cdot \omega_{X / B}\right)}{[k(P): k]},
$$

where $\omega_{X / B}$ is the canonical sheaf for $X$ over $B=\operatorname{Spec} R$, metrized with the canonical Arakelov metric (see [L 2, 4.3 and 4.5]). Note that $h_{K}$ is a Weil height for the canonical sheaf $K$ of $C$. As mentioned in the introduction, $d_{a}(P)$ is then defined as

$$
d_{a}(P):=\frac{\left(H_{P} \cdot\left(\omega_{X / B}+H_{P}\right)\right)}{[k(P): k]} .
$$

This definition closely resembles the definition of the arithmetic genus of a curve on a geometric surface. This resemblance explains some of the behavior of the arithmetic discriminant and makes it reasonable to expect that there might be a close relationship between diophantine questions on arithmetic surfaces and classical questions about geometric surfaces.

Let us also fix a horizontal divisor $F$ of degree 1 on $C$, so that we will have a fixed height function on $C$. In [V3], $F$ is taken to be the $\mathbb{Q}$-divisor $\omega_{X / B} /(2 g-2)$, but for our purposes it will be convenient to choose an actual divisor on $C$. We will want our divisor $F$ to have the property that $(2 g-2) F$ is linearly equivalent to $K_{C}$, so that heights taken with respect to our $F$ will differ from heights taken with respect to Vojta's $F$ by only a bounded constant. After extending $k$, we may assume that there there is some $Q$ in $C(k)$. Then, $K_{C}-(2 g-2) Q$ is in $\operatorname{Pic}^{0}(C)$. After extending $k$ again, we may assume that there is an element $Q^{\prime} \in \operatorname{Pic}^{0}(C)(k)$ such that $(2 g-2) Q^{\prime}=K_{C}-(2 g-2) Q$. Setting $F:=Q+Q^{\prime}$, we have a divisor with the desired properties. We define

$$
h(P):=h_{F}(P):=\frac{\left(H_{P} \cdot H_{F}\right)}{[k(P): k]},
$$

where $H_{F}$ is the horizontal divisor on $X$ corresponding to $F$. Henceforth, we will always drop the $A$ in the $h_{A}(P)$ in 0.0 .1 and use $h(P)$.

From the structure theorem for the Arakelov-Picard group of an arithmetic surface and the Arakelov adjunction formula ( $[\mathrm{L} 2$, Thm. 4.2.3] and [V 3, p. 791]), we know that

$$
\nu D_{P}^{2}=d_{a}(P)-h_{K}(P)-2 \nu h(P)+O(\nu)
$$

where $D_{P}$ is the element of the Picard-Arakelov group of $X$ corresponding to the $\mathbb{Q}$-divisor on $C \times_{k} \bar{k}$ of degree 0 given by

$$
\frac{1}{\nu}\left(P^{[1]}+\cdots+P^{[\nu]}-\nu F\right)
$$

where $P^{[i]}, i=1, \ldots, \nu$, are the conjugates of $P$ in $C(\bar{k})$. That this divisor is in fact $k$-rational follows from the fact that it is fixed by the Galois group of $\bar{k}$ over $k$. Now $D_{P}^{2}$ is always negative, by the positivity of the Néron-Tate height with respect to the $\Theta$-divisor of the Jacobian of $C$ ([L 1, Chapter 5]) and the fact that

$$
D_{P}^{2}=-2 h_{\mathrm{NT}}\left(D_{P}\right)
$$


(see [Ch, Thm. 5.1(ii)]). Thus, we obtain the following trivial upper bound

$$
d_{a}(P) \leq h_{K}(P)+2 \nu h(P)+O(\nu)
$$

for $d_{a}(P)$, as in [S-T, Section 2]. A lower bound on $d_{a}(P)$ is given by (0.0.1). We will refer to this lower bound as Vojta's inequality and we will always use it with the divisor $A$ taken to be $F$.

\section{A simple generalization of a corollary of Vojta}

In $\mathrm{A}-\mathrm{H}$, it is shown that a hyperelliptic curve of genus greater than 3 cannot be bi-elliptic (that is, admit a nonconstant map of degree 2 to an elliptic curve). Abramovich and Harris mention that the result is also a simple consequence of a diophantine result of Vojta and ask if it is possible to prove analogues of this corollary for maps to elliptic curves. We show that in fact this corollary has a simple generalization for maps to curves of any genus. First, let us reproduce the statement of the result of Vojta to which we are referring.

Corollary 2.1 (Cor. 0.2 of [V3]). Let $C$ be a curve of genus $g$ defined over a number field $k$, let $\nu$ be a positive integer, and let $f: C \longrightarrow \mathbb{P}^{1}$ be a dominant morphism. Assume that

$$
g-1 \geq(\nu-1) \operatorname{deg} f
$$

Then the set

$$
\{P \in C(k) \mid[k(P): k]=\nu \text { and } k(f(P))=k(P)\}
$$

is finite.

A weaker version of this result appeared in $[\mathrm{V} 2$, Theorem A]. The proof of the lemma below follows the proof given there.

Lemma 2.2. Let $C$ and $C^{\prime}$ be curves of genus $g$ and $g^{\prime}$, respectively, defined over a number field $k$, and let $f: C \longrightarrow C^{\prime}$ be a dominant $k$-morphism. Let $\mathcal{P}$ be a set of points $P \in C(\bar{k})$ of bounded degree satisfying the inequality

$$
d_{a}(f(P)) \leq h_{K_{C}^{\prime}}(f(P))+\operatorname{th}(f(P))+O(1),
$$

where $t$ is a real number such that $g-1>\left(t / 2+g^{\prime}-1\right) \operatorname{deg} f$. Then the set

$$
\{P \in \mathcal{P} \mid k(f(P))=k(P)\}
$$

is finite.

Proof. Let $P$ be a point in $\mathcal{P}$. Then, given any $\epsilon>0$, we have

$$
\begin{aligned}
d_{a}(P) & \leq d_{a}(f(P)) \\
& \leq h_{K_{C^{\prime}}}(f(P))+\operatorname{th}(f(P))+O(1) \\
& \leq\left(\left(2 g^{\prime}-2+t\right) \operatorname{deg} f+\epsilon\right) h(P)+O_{\epsilon}(1),
\end{aligned}
$$

using algebraic equivalence of divisors of equal degrees on $C$, the functorial property of heights, and the fact that $f^{*}$ sends divisors of degree 1 on $C^{\prime}$ to divisors of degree $\operatorname{deg} f$ on $C$. Vojta's inequality (and the fact that $K_{C}$ has degree $2 g-2$ ) then imply that

$$
(g-1) h(P) \leq\left(\left(t / 2+g^{\prime}-1\right) \operatorname{deg} f+\epsilon / 2\right) h(P)+O_{\epsilon}(1) .
$$


Choosing $\epsilon / 2>0$ smaller than $g-1-\left(t / 2+g^{\prime}-1\right) \operatorname{deg} f$ and applying Northcott's theorem (that there are only finitely algebraic points of bounded degree and bounded height) finishes the proof.

Proposition 2.3. Let $C$ and $C^{\prime}$ be curves of genus $g$ and $g^{\prime}$, respectively, defined over a number field $k$, let $\nu$ be a positive integer, and let $f: C \longrightarrow C^{\prime}$ be a dominant k-morphism. Assume that

$$
g-1>\left(\nu+g^{\prime}-1\right) \operatorname{deg} f
$$

The the set

$$
\{P \in C(\bar{k}) \mid[k(P): k]=\nu \text { and } k(f(P))=k(P)\}
$$

is finite.

Proof. By (1.0.6), we have the inequality $d_{a}(f(P)) \leq h_{K_{C^{\prime}}}(f(P))+2 \nu h(f(P))+$ $O(1)$ for all points $P$ in (2.3.2). Applying Lemma 2.2, with $\mathcal{P}$ taken to be the set of points $P$ on $C$ of degree $[k(P): k]=\nu$ and $t$ set equal to $2 \nu$, then gives the desired result.

Remark 2.4. Since the degree of the ramification divisor $R_{f}$ is

$$
\operatorname{deg} f=(2 g-2)-(\operatorname{deg} f-2) g^{\prime},
$$

the condition is (2.3.1) is equivalent to the condition

$$
\nu>\frac{\operatorname{deg} R_{f}}{2 \operatorname{deg} f} .
$$

In [S-T], the quantity $\left(\operatorname{deg} R_{f}\right)(\operatorname{deg} f)$ is used as a measure of how ramified a morphism is.

This proposition yields a simple, purely arithmetic proof of Castelnuovo's second genus-inequality in the special case that one of the curves being covered is elliptic. Castelnuovo's genus inequality follows fairly easily from the Hodge index theorem for surfaces; we present an alternate proof here that we will later modify, in Corollary 3.5 to obtain a stronger inequality that holds under certain conditions.

Corollary 2.5. Let $C$ be a curve that admits a map $\phi: C \longrightarrow C^{\prime} \times E$ that is birational onto its image, where $C^{\prime}$ is a curve and $E$ is a curve of genus 1 , and where $C, C^{\prime}, E$, and $\phi$ are all defined over any field of characteristic 0 . Let $f_{1}$ and $f_{2}$ denote the maps on $C$ obtained by composing $\phi$ with projection onto $C^{\prime}$ and $E$, respectively. Then

$$
g(C)-1 \leq\left(\operatorname{deg} f_{1}\right)\left(\operatorname{deg} f_{2}+g\left(C^{\prime}\right)-1\right)
$$

Proof. It will suffice to prove this in the case where all of the curves and morphisms are defined over a number field. The reduction to the case of number fields is standard and we only sketch it here. Since $C, C^{\prime}, E$, and $\phi$ can all be defined by a finite number of equations, they can all be defined over a field $L$ that is finitely generated over the rationals. Thus, we can write $L$ as $k\left(\alpha_{1}, \ldots, \alpha_{n}\right)$, where $k$ is a number field and the $\alpha_{i}$ are transcendental over $k$. Now, we can specialize each $\alpha_{i}$ to a value in $k$ without affecting the genus or nonsingularity of $C, C^{\prime}$, and $E$ or altering the degree of $\phi$. Hence, we have reduced to the case where everything is defined over a number field $k$.

The maps $f_{1}$ and $f_{2}$ yield inclusions of the function fields $k\left(C^{\prime}\right)$ and $k(E)$ into $k(C)$. Since $\phi$ is birational onto is image, $k(C)$ must be the compositum of $k\left(C^{\prime}\right)$ 
and $k(E)$ with respect to these inclusions. It follows that for any $P \in C(\bar{k})$, the residue field $k(P)$ must be the compositum of $k\left(f_{1}(P)\right)$ and $k\left(f_{2}(P)\right.$ ) (where the inclusions are the natural ones). Whenever $f_{2}(P) \in E(k)$, then, we must have $k(P)=k\left(f_{1}(P)\right)$. After extending the base field $k$, we may assume that there are infinitely many $Q \in E(k)$. The fiber $f_{2}^{-1}(Q)$ must contain a point $P \in C(\bar{k})$ with $[k(P): k] \leq \operatorname{deg} f_{2}$. Hence, there are infinitely many $P \in C(\bar{k})$ such that $[k(P): k] \leq \operatorname{deg} f_{2}$ and $k(P)=k\left(f_{1}(P)\right)$. Applying Proposition [2.3, we see that (2.5.1) must hold.

\section{Pull-BACKS OF $\Theta$ AND Zhang's RESUlt}

The bound we use to prove Proposition [2.3] is a fairly weak one. For many algebraic points $P$, the arithmetic discriminant is much smaller than

$$
h_{K}(P)+2[k(P): k] h(P) .
$$

To obtain improved bounds we need to control $D_{P}^{2}$, a formula for which was given in equation (1.0.5). It will be helpful, then, to take a close look at the heights with respect to $\Theta$-divisors of points in $\operatorname{Pic}^{0}(C)$.

Suppose we have a curve $C$ of genus $g \geq 2$ over a number field $k$. Let $C^{(d)}$ denote the $d$-th symmetric product of $C$ with itself. Points $P$ of degree $d$ give rise to rational points on $C^{(d)}$ by associating to $P$ the point in $C^{(d)}$ whose coordinates in $C^{d} \times_{k} \bar{k}$ are

$$
\left(P^{[1]}, \ldots, P^{[d]}\right),
$$

where $P^{[i]}$ are the conjugates of $P$ in $C(\bar{k})$ (see [Frey] and [Si]). That this point is in fact rational follows from the fact that the action of the symmetric group $S_{d}$ on $C^{d}$ identifies all of its $\bar{k}$ conjugates; hence it is fixed by $\operatorname{Gal}(\bar{k} / k)$. Now, there is a natural map from $C^{(d)}$ to $\operatorname{Pic}^{d}(C)$. The image of $C^{(d)}$ in $\operatorname{Pic}^{d}(C)$ is denoted as $W_{d}(C)$. More generally, one denotes as $W_{d}^{r}(C)$ the set of all divisor classes $D$ (under linear equivalence) of degree $d$ such that $h^{0}(C, D) \geq r+1$ (see $\mathrm{A}-\mathrm{C}-\mathrm{G}-\mathrm{H}$. Chapter 5]). When $r=0$, the superscript zero is omitted.

Of course, $\operatorname{Pic}^{d}(C)$ is isomorphic to the Jacobian of $C$, which we will denote by $J$. The group structure of $J$ makes it convenient for our purposes. We will also want to work on $C^{d}$ since its geometry is so simple. The maps we will be using are

$$
C^{d} \stackrel{s}{\longrightarrow} C^{(d)} \stackrel{\pi}{\longrightarrow} J .
$$

We will denote by $\phi$ the composition $\phi=\pi \circ s$. Note that $\pi$ is not defined naturally since it depends upon the isomorphism between $\operatorname{Pic}^{d}(C)$ and $J$ which depends on the choice of a degree $d$ line bundle on $C$. Our choice of degree $d$ line bundle does not matter, but, for convenience, let us choose $d F$. Now, since $-D_{P}^{2}$ is twice the Néron-Tate height, with respect to the $\Theta$-divisor of $J$, of the point corresponding to $D_{P}$ in $J$, as noted earlier, and Néron-Tate heights are quadratic (see [L 1, 5.3]), we have

$$
-D_{P}^{2}=\frac{2}{d^{2}} h_{\Theta}\left(\phi\left(P^{[1]}, \ldots, P^{[d]}\right)\right)+O(1) .
$$

It follows then from 1.0.3 that for points of degree $d$, we have

$$
d_{a}(P)-h_{K}(P)=2 d h(P)-(2 / d) h_{\phi^{*} \Theta}\left(\left(P^{[1]}, \ldots, P^{[d]}\right)\right)+O(1) .
$$

Thus, information about the structure of $\phi^{*}(\Theta)$ on $C^{d}$ should provide us with information about arithmetic discriminants of points. A result of Zhang ([Zh]) 
gives us information which shows that arithmetic discriminants of points for which $h^{0}\left(P^{[1]}+\cdots+P^{[d]}, C\right)=1$ are smaller than the arithmetic discriminants of families of points $P$ that correspond to the same $D_{P}$.

By Riemann-Roch, $h^{0}\left(C, P^{[1]}+\cdots+P^{[d]}\right)$ can be 1 only if $d \leq g$, so we restrict now to that case. When $d \leq g$, Riemann-Roch and semicontinuity of $h^{0}$ (see, for example, [Ha, 3.12]) show that the set $U$ of all $\left(P_{1}, \ldots, P_{d}\right)$ for which $h^{0}\left(C, P_{1}+\cdots+P_{d}\right)=1$ is a dense open subset of $C^{(d)}$. It is clear that the map $\phi$ is injective on $U$ and is therefore generically injective on $C^{(d)}$. With this terminology, here is Zhang's result.

Theorem $3.1([\overline{\mathrm{Zh}}])$. Let $p_{j}$ denote the projection of $C^{d}$ onto its $j$-th factor. There exist effective divisors $D_{1}, \ldots, D_{n}$ on $C^{d}$ and points $Q_{1}, \ldots, Q_{n}$ of $C$ such that

1. all $D_{i}$ are algebraically equivalent to $\phi^{*}(\Theta)$;

2. each $D_{i}$ includes an effective divisor $A_{Q_{i}}=\sum_{j=1}^{d} p_{j}^{*} Q_{i}$;

3. $\left(\bigcap_{i=1}^{n} D_{i}\right) \cap U=\emptyset$.

Remark 3.2. Poincaré's formula ([A-C-G-H, 1.5]) shows that homologically on $J$, one has

$$
w_{d} \cdot \Theta=(g+1-d) w_{d-1}
$$

where $w_{d}$ and $w_{d-1}$ are the homological cycle classes on $J$ corresponding to $W_{d}(C)$ and $W_{d-1}(C)$, respectively. This equality fails to hold in general for cycles on $W_{d}(C)$, up to algebraic equivalence, however. Indeed, if it held, one would see that (3.0.2) combined with Vojta's inequality would imply that $W_{2}(C)(k)$ was finite for any curve $C$ of genus greater than or equal to 4 . One sees this by noting that if (3.2.1) were correct up to algebraic equivalence and $W_{2}(C)(k)$ were infinite for a curve $C$ of genus $g \geq 4$, then $\phi^{*}(\Theta)$ would be algebraically equivalent to

$$
(g+1-2)\left(p_{1}^{*} F+p_{2}^{*} F\right)
$$

since $\pi: C^{(2)} \longrightarrow W_{2}(C)$ isomorphically (because $C$ cannot be hyperelliptic by V 3, Cor. 0.5]), which implies that for a quadratic point $P$ on $C$, the right hand side of (3.0.2) is

$$
(6-2 g+\epsilon) h(P)+O_{\epsilon}(1)
$$

for any $\epsilon>0$; this would violate Vojta's inequality. We know, however, that there exist curves of genus greater than 4 for which $W_{2}(C)(k)$ is infinite since there are bi-elliptic curves of every genus greater than or equal to 4 . We note that (3.2.1) fails to hold when $w_{d-1}$ and $w_{d} \cdot \Theta$ are considered as divisor classes on $W_{d}(C)$ not because of the difference between homological equivalence and algebraic equivalence (the two notions are the same for divisors and $w_{d-1}$ is a divisor on $W_{d}(C)$ - see $\mathrm{Fu}$. 19.3.1(i)]), but rather because the cycle map $i_{*}$ sending divisors on $W_{d}$ to cycles of codimension $(g+1-d)$ on $J$ fails to be injective on cycle classes up to algebraic equivalence. We also note that (3.2.1) does hold for divisor classes on $W_{d}(C)$ up to algebraic equivalence when $d=2$ and $C$ is hyperelliptic.

Corollary 3.3. For any $\epsilon>0$, there exists a constant $O_{\epsilon}(1)$ such that for all $P \in C(\bar{k})$ of degree $d$ with $h^{0}\left(C, P^{[1]}+\cdots+P^{[d]}\right)=1$, we have

$$
d_{a}(P) \leq h_{K}(P)+(2 d-2+\epsilon) h(P)+O_{\epsilon}(1) .
$$


Proof. If $P$ is a point of degree $d$, then all of the conjugates $P^{[1]}, \ldots, P^{[d]}$ of $P$ have the same height with respect to any fixed $k$-rational divisor, and the divisor $F$ is $k$-rational, so we may speak of the height of $h(P)$ without specifying a conjugate. Since the divisors corresponding to the $Q_{i}$ are all algebraically equivalent to $F$, we have for any $\epsilon^{\prime}>0$,

$$
\begin{aligned}
h_{A_{Q_{i}}}\left(P^{[1]}, \ldots, P^{[d]}\right) & =\sum_{j=1}^{d} h_{\left[Q_{i}\right]}(P) \\
& \geq \sum_{j=1}^{d} h(P)-\epsilon^{\prime} h(P)+O_{Q_{i}, \epsilon^{\prime}}(1) \\
& =d h(P)-\epsilon^{\prime} h(P)+O_{Q_{i}, \epsilon^{\prime}}(1) .
\end{aligned}
$$

Now, since $D_{i}-A_{Q_{i}}$ is effective, $h_{D_{i}-A_{Q_{i}}}$ is bounded from below away from the support of $D_{i}-A_{Q_{i}}([\mathrm{~L} 1$. Proposition 4.5.2]), so (3.3.2) implies that

$$
h_{D_{i}}\left(P^{[1]}, \ldots, P^{[d]}\right) \geq d h(P)-\epsilon^{\prime} h(P)+O_{D_{i}, \epsilon^{\prime}}(1),
$$

for points of $P$ outside of Supp $D_{i}$. Every such $P$ lies outside the support of $D_{i}-A_{Q_{i}}$ for some $i$ (by part (iii) of Theorem 3.1), so for each $P$ such that

$$
h^{0}\left(C, P^{[1]}+\cdots+P^{[d]}\right)=1,
$$

equation (3.3.3) applies to $P$ for some $i$. Furthermore, each $D_{i}$ is algebraically equivalent to $\phi^{*} \Theta$, so choosing $\epsilon^{\prime \prime}$ such that $(d / 2) \epsilon>\epsilon^{\prime \prime}>0$, we obtain

$$
h_{\phi^{*} \Theta}\left(P^{[1]}, \ldots, P^{[d]}\right) \geq d h(P)-\epsilon^{\prime \prime} h(P)+O_{\epsilon}^{\prime \prime}(1) .
$$

Applying (3.0.2) then gives us the desired inequality (3.3.1).

Note that this bound is strictly smaller than the formula obtained in S-T Section 4] for a family of algebraic points coming from pull-backs of rational points on $\mathbb{P}^{1}$. To save space, we will denote the divisor class of $P^{[1]}+\cdots+P^{[\nu]}$, where $1, \ldots, \nu$ are the conjugates of $P$, as $[P]$. Note that since it is an effective divisor class, $[P] \in W_{\nu}(C)$.

Proposition 3.4. Let $C$ and $C^{\prime}$ be curves of genus $g$ and $g^{\prime}$, respectively, defined over a number field $k$, let $\nu$ be a positive integer, and let $f: C \longrightarrow C^{\prime}$ be a dominant $k$-morphism. Suppose that

$$
g-1>\left(\nu+g^{\prime}-2\right) \operatorname{deg} f
$$

The the set

$$
\left\{P \in C(\bar{k}) \mid[k(P): k]=\nu, k(P)=k(f(P)), \text { and }[f(P)] \notin W_{\nu}^{1}(C)\right\}
$$

is finite.

Proof. For any point $P^{\prime} \in C^{\prime}(\bar{k})$ of degree $\nu$ for which $\left[P^{\prime}\right] \notin W_{\nu}^{1}\left(C^{\prime}\right)$ the inequality (0.0.1)

$$
d_{a}\left(P^{\prime}\right) \leq h_{K}\left(P^{\prime}\right)+2 \nu h\left(P^{\prime}\right)+O(1)
$$

can be replaced by (3.3.1)

$$
d_{a}\left(P^{\prime}\right) \leq h_{K}\left(P^{\prime}\right)+(2 \nu-2+\epsilon) h\left(P^{\prime}\right)+O_{\epsilon}(1) .
$$

Now, let $P$ be a point of degree $\nu$ on $C$ such that $P^{\prime}:=f(P)$ is also of degree $\nu$ and $\left[P^{\prime}\right] \notin W_{\nu}^{1}\left(C^{\prime}\right)$. Choosing a suitably small $\epsilon$ and applying Lemma 2.2 with $\mathcal{P}$ 
taken to be the set of these points $P^{\prime}$ and $t$ taken to be $2 \nu-2+\epsilon$, finishes the proof.

We immediately obtain better bounds on the genus of $C$ in Corollary 2.5 in certain cases. We need to introduce a small amount of notation. Given a map of curves $f: C \longrightarrow C^{\prime}$ we obtain push-forward maps $f_{*}: W_{d}(C) \longrightarrow W_{d}\left(C^{\prime}\right)$ and pull-back maps $f^{*}: W_{d}\left(C^{\prime}\right) \longrightarrow W_{(\operatorname{deg} f) d}(C)$. The fact that pushing forward and pulling back preserve linear equivalence (and hence give maps of Brill-Noether loci) is proved in [Fu, 1.4 and 1.7]).

Corollary 3.5. Let $C$ be a curve that admits a map $\phi: C \longrightarrow C^{\prime} \times E$ that is birational onto its image, where $C^{\prime}$ is a curve and $E$ is a curve of genus 1 , and where $C, C^{\prime}, E$, and $\phi$ are all defined over any field of characteristic 0 . Let $f_{1}$ and $f_{2}$ denote the maps induced by $\phi$ composed with projection onto $C^{\prime}$ and $E$, respectively. Suppose furthermore that $f_{1 *} f_{2}^{*}\left(\operatorname{Pic}^{1}(E)\right) \not \subset W_{\mathrm{deg} f_{2}}^{1}\left(C^{\prime}\right)$. Then

$$
g(C)-1 \leq\left(\operatorname{deg} f_{1}\right)\left(\operatorname{deg} f_{2}+g\left(C^{\prime}\right)-2\right) .
$$

Proof. As in Corollary 2.5 we may assume that all of the curves and morphisms are defined over a number field $k$. Since $f_{1 *} f_{2}^{*}\left(\operatorname{Pic}^{1}(E)\right) \not \subset W_{\nu}^{1}\left(C^{\prime}\right)$, the curve $f_{1 *} f_{2}^{*}\left(\operatorname{Pic}^{1}(E)\right)$ must intersect $W_{\nu}^{1}\left(C^{\prime}\right)$ in only finitely many points. Hence, we may apply Proposition 3.4 to all but finitely many points of the form $f_{1}(P) \in C^{\prime}(\bar{k})$ for $P \in C(\bar{k})$ which are in fibers $f_{2}^{-1}(Q)$ of rational points $Q \in E(k)$. Proceeding as in Corollary 2.5 then finishes the proof.

We note that this corollary sharpens the classical bounds of Castelnuovo (see A-C-G-H, Chapter 8, Ex. C-1]) in the special case under consideration, as one can see by comparing 2.5.1 with (3.5.1). We should note that when the genus of $C^{\prime}$ is not too small relative to $\operatorname{deg} f_{2}$ and $\operatorname{deg} f_{2}$ is a prime number, this bound can be improved further. The following proposition is an easy consequence of some results of $[\mathrm{A}-\mathrm{H}]$.

Proposition 3.6. Let $p$ be a prime number and suppose $E$ is an elliptic curve contained in $W_{p}(C)$ for a curve $C$ of genus greater than $p(p-1) / 2+1$. Suppose furthermore that $E$ is not contained in $W_{p-1}(C)+Q$ for any $Q \in C(\bar{k})$. Then there exists a map $\psi: C \longrightarrow E^{\prime}$ from $C$ to an elliptic curve $E^{\prime}$ for which $E=$ $\psi^{*}\left(\operatorname{Pic}^{1}\left(E^{\prime}\right)\right)$.

Proof. By Theorem 2 and Lemma 3 of $\left[\mathrm{A}-\mathrm{H}\right.$, either $E$ is contained in $W_{p}^{1}(C)$ or there exists a map $\phi: C \longrightarrow C^{\prime}$ of degree greater than 1 to a curve $C^{\prime}$ and an elliptic curve $E^{\prime} \subset W_{d^{\prime}}\left(C^{\prime}\right)$, where $d^{\prime}=p /(\operatorname{deg} \phi)$, such that $E=\phi^{*} E^{\prime}$. Since $p$ is prime this can only happen when $\operatorname{deg} \phi=p$. This means that $C^{\prime}$ must be an elliptic curve since $W_{1}\left(C^{\prime}\right)$ contains an elliptic curve.

Remark 3.7. Abramovich and Harris assume throughout their paper $\mathrm{A}-\mathrm{H}$ ] that their abelian subvarieties of Jacobians are "minimally" embedded in the sense that they are not contained in $W_{d-1}(C)+Q$ for any $Q \in C(\bar{k})$ (see the beginning of Section 2 of the paper). This implies, via Riemann-Roch, that these abelian subvarieties are not contained in $W_{d}^{1}(C)$, which is stated as an assumption in A-H. Lemma 3].

Corollary 3.8. Let $C$ be a curve that admits a map $\phi: C \longrightarrow C^{\prime} \times E$ that is birational onto its image, where $C^{\prime}$ is a curve and $E$ is a curve of genus 1 , and 
where $C, C^{\prime}, E$, and $\phi$ are all defined over any field of characteristic 0 . Let $f_{1}$ and $f_{2}$ denote the maps induced by $\phi$ composed with projection onto $C^{\prime}$ and $E$, respectively. Suppose furthermore that $f_{1 *} f_{2}^{*}\left(\operatorname{Pic}^{1}(E)\right) \not \subset W_{\operatorname{deg} f_{2}}^{1}\left(C^{\prime}\right)$, that $g\left(C^{\prime}\right)>$ $\left(\operatorname{deg} f_{2}\right)\left(\operatorname{deg} f_{2}-1\right) / 2+1$, and that $\operatorname{deg} f_{2}$ is a prime number. Then $f_{1}$ is étale and $g(C)=\left(\operatorname{deg} f_{1}\right)\left(g\left(C^{\prime}\right)-1\right)+1$.

Proof. As in Corollaries 2.5 and 3.5. we may assume that all of the curves and morphisms are defined over a number field $k$. In order to apply Proposition 3.6 to $f_{1 *} f_{2}^{*}\left(\operatorname{Pic}^{1}(E)\right)$, we need to show that $f_{1 *} f_{2}^{*}\left(\operatorname{Pic}^{1}(E)\right)$ is not contained in $W_{\operatorname{deg} f_{2}-1}(C)+Q$ for any $Q \in C(\bar{k})$. Since $f_{1 *} f_{2}^{*}\left(\operatorname{Pic}^{1}(E)\right)$ is not contained in $W_{\mathrm{deg} f_{2}}^{1}(C)$ all but finitely many of the elements of $f_{1 *} f_{2}^{*}\left(\mathrm{Pic}^{1}(E)\right)$ have unique representations as effective divisors $D$ of degree $f_{2}$ on $C$. Hence, we need only show that there is no $Q \in C(\bar{k})$ contained in the support of all of these $D$. It is not hard to see that this is the case, since by the definitions of $f_{1 *}$ and $f_{2}^{*}$, each $D$ can be written as a sum

$$
\sum_{f_{2}\left(P^{\prime}\right)=P} f_{1}\left(P^{\prime}\right)
$$

for some $P \in E(\bar{k})$; it follows that any $Q \in C(\bar{k})$ occurs in at most $\operatorname{deg} f_{1}$ of the divisors in $f_{1 *} f_{2}^{*}\left(\operatorname{Pic}^{1}(E)\right)$ that are not in $W_{\operatorname{deg} f_{2}}^{1}(C)$. Applying Proposition 3.6, see that there exists a map $\psi: C^{\prime} \longrightarrow E^{\prime}$ to an elliptic curve $E^{\prime}$ such that $f_{1 *} f_{2}^{*}\left(\operatorname{Pic}^{1}(E)\right)=\psi^{*}\left(\operatorname{Pic}^{1}\left(E^{\prime}\right)\right)$. We note that $\psi^{*}$ must be an isomorphism onto its image since otherwise it would have degree greater than 1 , which is not possible since $\psi^{*}\left(\operatorname{Pic}^{1}\left(E^{\prime}\right)\right)$ is not contained in $W_{p}^{1}\left(C^{\prime}\right)$; similarly, $f_{2}^{*}$ must be an isomorphism onto its image. It follows that $f_{2 *}$ induces an isogeny $g: E \longrightarrow E^{\prime}$ such that $\psi f_{1}=g f_{2}$. After extending the base field of $E$ we may assume that $E(k)$ is infinite and that there are therefore infinitely many $Q \in C(\bar{k})$ such that $f_{2}(Q) \in E(k)$. This means that for $P=f_{1}(Q)$, we must have $\psi(P) \in E^{\prime}(k)$, since $g f_{2}(Q) \in E^{\prime}(k)$. By the Chevalley-Weil theorem ( $\mathrm{V} 2$, Thm. 5.1.6]), we have

$$
d_{a}(P) \leq d_{a}(\psi(P))+h_{R_{\psi}}(P)+O(1),
$$

where $R_{\psi}$ is the ramification divisor of $\psi$. If $\psi(P) \in E^{\prime}(k)$, an application of Riemann-Hurwitz then yields

$$
d_{a}(P) \leq h_{K_{C^{\prime}}}(P)+O(1),
$$

since $d_{a}(\psi(P)) \leq O(1)$ and $K_{E}$ is trivial. Now, as in Corollary [2.5] it is clear that $k(Q)=k(P)$ (since $f_{2}(Q) \in E(k)$ ). Lemma 2.2 implies that $g(C)-1=$ $\left(\operatorname{deg} f_{1}\right)\left(g\left(C^{\prime}\right)-1\right)$, which means that $f_{1}$ is étale, by the Riemann-Hurwitz formula.

Remark 3.9. It would be interesting to have results similar to Corollaries 3.5 and 3.8 in the case that $E$ is replaced by a curve $C_{2}$ of arbitrary genus $g_{2}$ and

$$
f_{1 *} f_{2}^{*}\left(\operatorname{Pic}^{g_{2}}\left(C_{2}\right)\right) \not \subset W_{g_{2}\left(\operatorname{deg} f_{2}\right)}^{1}\left(C^{\prime}\right) .
$$

One may be able to use results of [Frey to apply the method of Corollary 3.5 to obtain some generalizations but only under the more restrictive hypothesis that $W_{g_{2}\left(\operatorname{deg} f_{2}\right)}^{1}\left(C^{\prime}\right)$ is empty. It may also be possible to prove results along these lines using a direct analysis of the Néron-Severi group of $C^{\prime} \times C_{2}$. 
The most obvious way for points of degree $\nu$ or less to arise on a curve is via nonconstant maps of degree $\nu$ or less to $\mathbb{P}^{1}$ or elliptic curves. We saw in the previous corollary that points $P$ in fibers over rational points of elliptic curves have "smallest possible" arithmetic discriminants; that is to say, they satisfy $d_{a}(P)=$ $h_{K}(P)+O(1)$. On the other hand, if one has a family of points $P$ all of which form fibers over rational points of $\mathbb{P}^{1}$ with respect to a fixed map to $\mathbb{P}^{1}$, then all of the divisor classes $[P]$ are linearly equivalent and all of the $P$ give rise to the same $D_{P}$ in the notation of (1.0.3). This implies that all of these $P$ satisfy the equality $d_{a}(P)=h_{K}(P)+2[k(P): k] h(P)+O(1)$. These points thus have "largest possible" arithmetic discriminants. It is natural to ask if there are points which have arithmetic discriminants which are neither smallest possible nor largest possible. In the next section, we examine points of degree 2 on curves of genus 2 and demonstrate that there are such families of points on bi-elliptic curves of genus 2. In the process of doing this, we will obtain a curve that admits two covers and which has genus as small as is permitted by Corollary 3.8

\section{Quadratic points on Curves of Genus 2}

Throughout this section $C$ will be a curve of genus 2 defined over a number field. By $\mathrm{A}-\mathrm{C}-\mathrm{G}-\mathrm{H}$, Chapter 6, Ex. I-1], $C^{(2)}$ is the blow-up of $\operatorname{Pic}^{2}(C)$ at the point corresponding to the canonical divisor of $C$. We also know that a $\Theta$ divisor on $J$ is simply the image of $C$ in $J$ with respect to an injection of $C$ into $J$. It is fairly easy then to describe the structure of $\phi^{*} \Theta$. We are only interested in determining this structure up to algebraic equivalence, so let us fix the injection of $C$ into $J$ by choosing a ramification point $Q$ (which we may take to be $k$-rational by extending $k$ ) of the hyperelliptic map on $C$ and defining $i: C \hookrightarrow J$ by $i(P)=[P-Q]$ and treat the divisor class of $i(C)$ as the $\Theta$-divisor of $J$; these two divisor classes are algebraically equivalent by Riemann's Theorem ( $\mathrm{A}-\mathrm{C}-\mathrm{G}-\mathrm{H}$, p. 27]).

Since the map $\pi: C^{(2)} \longrightarrow J$ is a blow-down at a single point, $\pi^{*} \Theta$ is simply $\tilde{\Theta}+m D$ where $\tilde{\Theta}$ is the strict transform of $\Theta, D$ is the exceptional divisor of $\pi$, and $m$ is the multiplicity of $\Theta$ at (0) (since (0) is the image of $K_{C}$ in $J$ via the isomorphism we chose between $J$ and $\operatorname{Pic}^{2}(C)$ ). It is easy to verify that $m=1$, because $\Theta$ clearly contains (0) and $\Theta$ is nonsingular.

Now, $\phi(\{Q\} \times C)=\phi(C \times\{Q\})=\Theta$ and $\phi$ is a map of degree 2 away from the point (0); hence, $s^{*} \tilde{\Theta}=\{Q\} \times C+C \times\{Q\}$. One also sees that if $\sigma$ is the hyperelliptic involution of $C$, then all points in $C^{(2)}(\bar{k})$ of the form $(P, \sigma P)$ have the property that $P+\sigma P \sim K_{C} \sim 2 Q$; hence, they all map to $(0)$ in $J$. The set of all such points forms a curve, which we call $\Delta_{\alpha}$ (it is the graph of $\sigma$ ). Since there is one rational curve in $C^{(2)}$ corresponding to a unique nontrivial linear equivalence class of divisors, $s\left(\Delta_{\alpha}\right)$ must be this curve. This curve is also the exceptional divisor $D$ of $\pi$ as noted earlier. Hence, it is clear that $\phi^{*} \Theta=\Delta_{\alpha}+\{Q\} \times C+C \times\{Q\}$.

There does not seem to be much we can say about the heights of points in $C^{(2)}$ with respect to $\Delta_{\alpha}$, in general. When $J$ is not simple, however, we can say a lot more. Under these circumstances, $J$ contains an elliptic curve; in fact, every point on $J$ lies on some elliptic curve in $J$. Since the algebraic equivalence class of a divisor on a curve is determined completely by the divisor's degree, we can obtain a great deal of information about arithmetic discriminants of points on $J$ simply by calculating the intersections of $\phi^{*} \Theta$ with pull-backs of elliptic curves on $J$. It is easy to see that whenever the Jacobian of a curve $C$ of genus 2 is not simple, 
$C$ must admit a nonconstant map to an elliptic curve (one composes an injection of $C$ into $J$ with the map from $J$ onto an elliptic curve). Our calculations will be particularly simple when there exists a map $\psi: C \longrightarrow E$ of degree 2 to an elliptic curve, so let us now restrict to that case. We see in this case that there is a nontrivial involution $\beta$ of $C$ such that $\psi \cdot \beta=\psi$. Letting $\Delta_{\beta}$ be the graph of this automorphism in $C^{2}$, we see that $s\left(\Delta_{\beta}\right)$ is a curve in $C^{(2)}$ isomorphic to $E$. Let us call this curve $E$, in a mild abuse of notation. Let us also denote its image $\pi(E)$ in $J$ as $E^{\prime}$. The following lemma will be useful.

Lemma 4.1. With notation as above, $E \cdot D=1$.

Proof. Since $E$ and $J$ are abelian varieties the inclusion of $E$ into $J$ must factor as a homomorphism composed with a translation. It follows that $E$ and any nontrivial translate of $E$ are distinct cosets of a subgroup and hence do not intersect. All translates of a divisor are numerically equivalent, so we have

$$
E^{\prime 2}=0 \text {. }
$$

We may also calculate the self-intersection of $E$ by using the self-intersection of $\Delta_{\beta}$. Applying the adjunction formula for surfaces ([Ha, Proposition 5.1.5]) to $\Delta_{\beta}^{2}$ yields

$$
\begin{aligned}
\Delta_{\beta}^{2} & =2 g\left(\Delta_{\beta}\right)-2-K_{C^{2}} \cdot \Delta_{\beta} \\
& =2-2[\{Q\} \times C+C \times\{Q\}] \cdot \Delta_{\beta} \\
& =-2 .
\end{aligned}
$$

Now, using basic properties of pull-backs of intersections ([Fu Theorem 6.2]), we have $\Delta_{\beta}^{2}=\left(s^{*} E\right)^{2}=s^{*}\left(E^{2}\right)=2\left(E^{2}\right)$. Hence, $E^{2}=-1$. This means that $E \cdot D=1$. To see this one notes that $\pi^{*} E^{\prime}=E+m D$ for some nonnegative $m$ and that from the projection formula and (4.1.1), we have $E+m D \cdot E=E^{\prime 2}=0$, which implies that $E^{2}+m(E \cdot D)=0$. This can only happen when $E \cdot D=1$ and $m=1$.

It is easy to calculate $d_{a}(P)$ when $P$ is a quadratic point in $C(\bar{k})$ for which the conjugate pair $\left(P^{[1]}, P^{[2]}\right)$ lies in $E$. In this case, the Chevalley-Weil theorem gives $d_{a}(P)=h_{K}(P)+O(1)$ (as in the proof of Corollary 3.8). We are also able to use Lemma 4.1 to calculate the arithmetic discriminants of the quadratic points on $C$ which are not pull-backs of rational points of $E$. We see that every rational point on $J$ lies on some translate $E^{\prime}+x$ of $E^{\prime}$, which we denote as $E_{x}^{\prime}$. If $x \notin E^{\prime}$, then $E^{\prime}+x$ does not pass through (0). It follows then that $\pi^{*} E_{x}^{\prime}$ is irreducible and does not intersect $D$. Note that this means that $\phi^{*} E_{x}^{\prime}$ is irreducible, since if it had two irreducible components, each would have to admit a degree 1 map to $E$ yet cover $C$, which is impossible since $g(C)>g(E)$. Let us denote the (possibly singular) curve corresponding to $\phi^{*} E_{x}^{\prime}$ as $C_{x}^{\prime}$.

Proposition 4.2. For a fixed $x \in J(k)$, all of the points $P$ for which the conjugate pair $\left(P^{[1]}, P^{[2]}\right)$ lies on $C_{x}^{\prime}$ satisfy

$$
d_{a}(P)=h_{K}(P)+2 h(P)+O_{x}(1) .
$$

Proof. Whenever $P^{[1]}$ and $P^{[2]}$ are the conjugates of a quadratic point on $C$, plugging $d=2$ into 3.0 .2 yields

$$
d_{a}(P)=h_{K}(P)+4 h(P)-h_{\phi^{*} \Theta}\left(P^{[1]}, P^{[2]}\right)+O(1) .
$$


Now, $C_{x}^{\prime}$ does not intersect $\Delta_{\alpha}$ (because $E_{x}^{\prime}$ does not pass through $\left.(0)\right)$, so $C_{x}^{\prime} \cdot \phi^{*} \Theta=$ $C_{x}^{\prime} \cdot(\{Q\} \times C+C \times\{Q\})$. This implies that

$$
h_{\phi * \Theta}\left(P_{1}, P_{2}\right)=h_{[Q]}\left(P_{1}\right)+h_{[Q]}\left(P_{2}\right)+O_{x}(1)=2 h_{[Q]}(P)+O(1)
$$

holds when $\left(P_{1}, P_{2}\right) \in C_{x}^{\prime}$. Applying this to conjugate pairs $\left(P^{[1]}, P^{[2]}\right) \in C_{x}^{\prime}$ and combining with (4.2.2) yields

$$
d_{a}(P)=h_{K}(P)+4 h(P)-2 h_{[Q]}+O_{x}(1) .
$$

Since $2 Q \sim K_{C}$, the height functions $h_{[Q]}$ and $h$ are the same up to a bounded constant, this gives the desired result.

Let $\tilde{C}_{x}$ denote the desingularization of $C_{x}^{\prime}$. Since $\tilde{C}_{x}$ admits maps of degree 2 to $E$ and to $C$ (which it inherits from $C_{x}^{\prime}$ via the natural map $\tilde{C}_{x} \longrightarrow C_{x}^{\prime}$ ), it is clear that there is a map $\tilde{C}_{x} \longrightarrow C \times E$ that is birational onto its image. By Corollary 3.5 (which we may apply since $W_{2}^{1}(C)$ is a single point), the genus of $\tilde{C}_{x}$ must be at most 5. We see now that it is exactly 5 , in general, and that Corollary 3.5 is therefore sharp in this case. First, we need to introduce maps on $J$ and $C$. Note that modding out by $E^{\prime}$ gives us a map from $J$ to an elliptic curve. Let us call this elliptic curve $\tilde{E}$ and let us denote as $\rho$ the natural map $\rho: J \longrightarrow \tilde{E}$ obtained by modding out by $E^{\prime}$. Let us also denote as $\gamma$ the map on $C$ obtained by composing $\rho$ with the inclusion $i: C \longrightarrow J$ defined earlier. We find the degree of $\gamma$ below.

Lemma 4.3. The map $\gamma: C \longrightarrow \tilde{E}$ has degree 2 .

Proof. It will suffice to show that $E^{\prime} \cdot \Theta=2$, since this implies that the fiber of $\gamma$ over the identity of $\tilde{E}$ has degree 2 . To show this, we begin by calculating $\Delta_{\beta} \cdot \Delta_{\alpha}$, again using the projection formula. We obtain

$$
\Delta_{\beta} \cdot \Delta_{\alpha}=s^{*} E \cdot \Delta_{\alpha}=E \cdot s_{*} \Delta_{\alpha}=E \cdot 2 D=2 .
$$

We now have all the information we need to calculate $\Delta_{\beta} \cdot \phi^{*} \Theta$. We have

$$
\Delta_{\beta} \cdot \phi^{*} \Theta=\Delta_{\beta} \cdot\left(\{Q\} \times C+C \times\{Q\}+\Delta_{\alpha}\right)=4 .
$$

Since $\phi_{*} \Delta_{\beta}=2 E^{\prime}$, we obtain $E^{\prime} \cdot \Theta=2$ via the projection formula.

Now, we are able to show that for general $x, \tilde{C}_{x}$ has genus 5 .

Proposition 4.4. The curves $\tilde{C}_{x}$ have genus 5 for all but finitely many values of $\rho(x)$.

Proof. We begin by describing each curve $C_{x}^{\prime}$ as a fiber product. We observe that $C_{x}^{\prime}$ admits two maps to $C$ induced by the two projections from $C \times C$ onto $C$. Let us denote these two maps from $C_{x}^{\prime}$ to $C$ as $p_{1}$ and $p_{2}$. Now, when $\rho(x) \neq 0, C_{x}^{\prime}$ is simply the set of points $\left(P_{1}, P_{2}\right)$ of $C^{2}$ for which $\gamma\left(P_{1}\right)=-\gamma\left(P_{2}\right)+\rho(x)$. This means that the following diagram is a fiber square

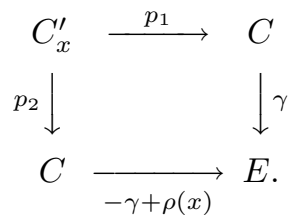

Note that the ramification locus of $\gamma$ in $E$ consists of two points; let us call them $Q_{1}$ and $Q_{2}$. If $\rho(x)$ is not equal to $2 Q_{1}, 2 Q_{2}$, or $Q_{1}+Q_{2}$, then the ramification loci of $\gamma$ and $-\gamma+\rho(x)$ are disjoint. This implies that $C_{x}^{\prime}$ has no singularities and is 
therefore equal to its desingularization $\tilde{C}_{x}$. It also implies that $p_{1}$ has exactly four ramification points, which means the genus of $\tilde{C}_{x}$ is 5 , by the Riemann-Hurwitz formula.

Remark 4.5. It is easy to see that the diagram

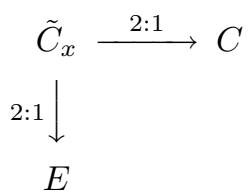

does not split in the sense of $\mathrm{Ma}$; that is to say, there is no way of choosing a base curve $B$ so that $C$ and $E$ admit maps to $B$ of degree 2 or less extending the diagram to a commutative square. Martens demonstrates that there is a curve of genus 4 which admits maps of degree 2 to curves of genus 1 and 2 which fail to split. Our curves $\tilde{C}_{x}$ also have genus 4 in the case that $\rho(x)=2 Q_{1}$ and $2 Q_{1} \neq 2 Q_{2}$, where $Q_{1}$ and $Q_{2}$ are defined as in the proof of Proposition 4.4, and it turns out that Martens' curve of genus 4 also arises in this manner. We also note that when $2 Q_{1}=2 Q_{2}$ and $\rho(x)=2 Q_{1}=2 Q_{2}$, the curve $\tilde{C}_{x}$ has genus 3 and the map from $\tilde{C}_{x}$ to $C$ is étale. In this case, the image in $C$ of the quadratic points on $\tilde{C}_{x}$ have the smallest field discriminants $d(P)$ permitted by Vojta's conjecture although their arithmetic discriminants are not "smallest possible." This is a phenomenon that also occurs in [S-T, Sections 3 and 4$]$.

In closing, we would like to make a few general statements about arithmetic discriminants of quadratic points on curves. A curve $C$ of genus 2 may have a non-simple Jacobian without being bi-elliptic. The subject of curves of genus 2 that cover elliptic curves is, in fact, quite complex (see, for example, Kani]). While some of the work done here carries over to curves of genus 2 that are not bi-elliptic but have split Jacobians, we are not able to answer some basic questions about the arithmetic discriminants of quadratic points on such curves; for example, it is not clear whether or not Vojta's inequality is sharp for quadratic points on such curves. Arithmetic discriminants of quadratic points on curves of genus 2 with simple Jacobians seem to be even more difficult to control. On the other hand, arithmetic discriminants of quadratic points on curves of genus greater than 2 are easy to classify. When $C$ is a curve of genus greater than 2, the Brill-Noether locus $W_{2}(C)$ cannot be an abelian variety. It follows from Faltings' theorem for subvarieties of an abelian variety ( $[\mathrm{Fa} 1])$ that $W_{2}(C)$ can contain infinitely many rational points only if it contains an elliptic curve. As observed in $[\mathrm{A}-\mathrm{H}]$ and $\underline{\mathrm{H}-\mathrm{S}}$, this means that a curve $C$ of can have infinitely many quadratic points only if it is covered by a bi-elliptic or hyperelliptic curve. The main result of $\mathrm{Ma}$ then implies that on a curve $C$ of genus greater than 2 , all of the quadratic points are obtained via bi-elliptic or hyperelliptic maps on $C$ itself.

\section{REFERENCES}

[A-H] D. Abramovich and J. Harris, Abelian varieties and curves in $W_{d}(C)$, Comp. Math. 78 (1991:2), 227-238. MR 92c:14022

[A-C-G-H] E. Arbarello, M. Cornalba, P.A. Griffiths, and J. Harris, Geometry of algebraic curves I, Springer-Verlag, New York, 1985. MR 86h:14019

[Ar] M. Artin, Lipman's proof of resolution of singularities for surfaces, in Arithmetic geometry (edited by G. Cornell and J. Silverman), Springer-Verlag, New York, 1986, 267-288. MR 89b:14029 
[Ch] T. Chinburg, An introduction to Arakelov intersection theory, in Arithmetic geometry (edited by G. Cornell and J. Silverman), Springer-Verlag, New York, 1986, 289-308. MR 89b:14029

[D-F] O. Debarre and R. Fahlaoui, Abelian varieties in $W_{d}^{r}(C)$ and points of bounded degree on algebraic curves, Comp. Math., 88 (1993), 235-249. MR 94h:14028

[Fa 1] G. Faltings, Diophantine approximation on abelian varieties, Ann. of Math. 133 (1991:2), 549-576. MR 93d:11066

[Fa 2] G. Faltings, The general case of S. Lang's conjecture, in Christante, V. and Messing, W. (eds.), Barsotti symposium in algebraic geometry, Perspectives in Mathematics 15, Academic Press, San Diego, Calif., 1994, 175-182. MR 95m:11061

[Frey] G. Frey, Curves with infinitely many points of fixed degree, Israel J. Math., 85 (1994), 79-83. MR 94m:11072

[Fu] W. Fulton, Intersection Theory, Springer-Verlag, Berlin, 1984. MR 85k:14004

$[\mathrm{H}-\mathrm{S}] \quad$ J. Harris and J. Silverman, Bielliptic curves and symmetric products, Proc. Amer. Math. Soc., 112 (1991:2), 347-356. MR 91i:11067

[Ha] R. Hartshorne, Algebraic geometry, Springer-Verlag, Graduate Texts in Mathematics, vol. 52, New York, 1977. MR 57:3116

[Kani] E. Kani, The number of curves of genus 2 with elliptic differentials, J. Reine Angew. Math., 485 (1997), 93-121. MR 98g:14025

[L 1] S. Lang, Fundamentals of diophantine geometry, Springer-Verlag, New York, 1983. MR 85j:11005

[L 2] S. Lang, Introduction to Arakelov theory, Springer-Verlag, New York, 1988. MR 89m:11059

[Ma] G. Martens, On coverings of elliptic curves, in Algebra and number theory (Essen, 1992), de Gruyter, Berlin, 1994, 137-151. MR 95e:14024

[Si] J. Silverman, Rational points on symmetric products of a curve, Amer. J. Math. 113 (1991), 471-508. MR 92m:11060

[S-T] X. Song and T. J. Tucker, Dirichlet's Theorem, Vojta's inequality, and Vojta's conjecture, Comp. Math. 116 (1999:2), 219-238. MR 2000d:11085

[Tu] T. J. Tucker, Generalizations of Hilbert's irreducibility theorem, preprint.

[V 1] P. Vojta, Diophantine approximations and value distribution theory, Lecture Notes in Math., vol. 1239, Springer-Verlag, New York, 1987. MR 91k:11049

[V 2] P. Vojta, Arithmetic discriminants and quadratic points on curves, in Arithmetic algebraic geometry (Texel, 1989), Progr. Math. 89, Birkhäuser Boston, Boston, MA, 1991, 359-376. MR 92j:11059

[V 3] P. Vojta, A generalization of theorems of Faltings and Thue-Siegel-Roth-Wirsing, J. Amer. Math. Soc., 5 (1992:4), 763-804. MR 94a:11093

[Zh] S. Zhang, Note to G. Frey, 1994.

Department of Mathematics, University of California-Berkeley, Berkeley, CaliforNIA 94720

E-mail address: song@math.berkeley.edu

Department of Mathematics, University of Georgia, Athens, Georgia 30602

E-mail address: ttucker@math.uga.edu 